\title{
Effect of Ultra-Soft and Soft Toothbrushes on the Removal of Plaque and Tooth Abrasion
}

\author{
Moon-Jin Jeong ${ }^{1}$, Han-A Cho, Su-Yeon Kim, Ka-Rim Kang, Eun-Bin Lee, Ye-Ji Lee, Jung-Hyeon Choi, \\ Ki-Sung Kil ${ }^{1}$, Myoung-Hwa Lee ${ }^{1}$, Soon-Jeong Jeong ${ }^{2}$, and Do-Seon Lim ${ }^{\dagger}$ \\ Department of Dental Hygiene, College of Health Science, Eulji University, Seongnam 13135, \\ ${ }^{1}$ Department of Oral Histology and Biology, School of Dentistry, Chosun University, Gwangju 61452, \\ ${ }^{2}$ Department of Dental Hygiene, College of Health Sciences, Youngsan University, Yangsan 50510, Korea
}

To improve the oral health status of Korean people, it is necessary to encourage proper oral hygiene management habits, such as toothbrushing, through appropriate health promotion techniques. Therefore, the purpose of this study was to evaluate the removal of plaque and tooth abrasion using ultra-soft (filament $0.11 \sim 0.12 \mathrm{~mm}$ ) and soft toothbrushes for toothbrushing. The plaque removal was performed using a dentiform and Arti-spray, and the Patient Hygiene Performance (PHP) index was calculated as the sum total score divided by the total number of surfaces. In the abrasivity experiment, according to the number of brushings, a micro Vickers hardness tester was used, and a sample in the range of $280 \sim 380$ Vickers hardness number was selected. The number of toothbrushing stroke were 1,800 (2 months), 5,400 (6 months), 10,800 (12 months), and 21,600 (24 months). The tooth abrasion was measured using a scanning electron microscope. Statistical analysis was performed using IBM SPSS Statistics 22.0 and a p-value $<0.05$ was considered significant. According to the results, there was no statistically significant difference in the degree of plaque removal between ultra-soft and soft toothbrushes. The difference in tooth abrasion between before and after toothbrushing was found to be greater with the soft toothbrushes than with the ultra-soft toothbrushes. Therefore, the ultra-soft toothbrush not only lowers tooth damage by reducing tooth abrasion, but also shows a similar ability to remove plaque as soft toothbrushes.

Key Words: Dental plaque, Tooth abrasion, Toothbrushing

\section{Introduction}

Oral health is an important factor in systemic health and quality of life $^{1)}$. Oral diseases are diffuse, chronic, irreversible, and progressive. Unlike other diseases, they are preventable; therefore, the early detection of risk factors should be prioritized to prevent the progression of oral disease ${ }^{2}$. Toothbrushing, which is a method of oral disease prevention, removes bacteria related to dental caries and periodontal diseases, and is considered the most reliable method of plaque removal ${ }^{3,4)}$. The Health Plan 2020 (2016 2020) defines proper toothbrushing as the most appropriate health promotion behavior that can manage both dental caries and periodontal disease, and states that it is necessary to encourage habitual and appropriate oral health management in line with various health promotion projects that are being developed for different life cycle $^{5)}$. There has been a consistent discussion concerning oral health promotion projects worldwide and a demand for programs for the prevention of oral diseases and health promotion ${ }^{1)}$. Based on the OECD (Organization for Economic Cooperation and Development) health index, South Korea is ranked 24th of 28 OECD countries in oral health status ${ }^{6}$. Therefore, it is necessary to pay attention to exploring various ways to promote oral health ${ }^{6}$.

Toothbrushes for different ages and with different functions have been developed and have been useful for personal oral health management by individuals. The 
factors that affect plaque removal include toothbrush $\operatorname{design}^{4)}$, toothbrushing method ${ }^{4)}$, an individual's toothbrushing ability ${ }^{7}$, frequency, and duration of tooth brushing ${ }^{4,7)}$, bristle shape ${ }^{7)}$, material, length, diameter, number of bristles, and arrangement ${ }^{8)}$. Choosing the appropriate bristle type is especially important because bristles come in contact with the teeth and gums. Bristles are classified as hard, regular, and soft/ultra-soft according to the filament stiffness grade of the brush head ${ }^{9,10)}$. Study results on the effect of soft bristles on the cleaning ability of toothbrush and tooth abrasion have been consistently reported. Most commercially available toothbrushes have mild to medium-hard bristles ${ }^{11)}$. In response to teeth and gum weakening in modern humans, soft tooth brushes emerged and expanded the range of choice for customers. Soft toothbrushes can brush the boundaries between the teeth and gums in the presence of a fixed prosthesis more effectively and can reduce gingival recession and abrasion. Since hard toothbrushes can cause enamel and dentin abrasion, and can weaken teeth covered in a prosthesis, ultra-soft toothbrushes are recommended ${ }^{12)}$. Ultra-soft toothbrushes that use a vibration motion to clean the teeth and gingival margin are also recommended for the management of diabetic patients' oral health ${ }^{13)}$. Ultra-soft toothbrushes with bristles arranged in a conical shape have been reported to remove dental plaques in surgical sites following surgery more quickly than regular toothbrushes ${ }^{14)}$. They are also recommended for patients with mouth pain or inflammation ${ }^{15,16)}$. Ultra-soft toothbrushes are used as toothbrushes for children, and as professional toothbrushes (e.g., TePe, professional care toothbrushes, etc. ${ }^{15)}$. Use of these toothbrushes by patients with gingiva-related problems has also been reported ${ }^{17,18)}$.

The easiest method to improve oral hygiene is toothbrushing. Studies have focused on the effectiveness of toothbrushes in plaque removal and their convenience of use. However, the choice and use of a toothbrush depends on an individual's preference. The choice of a toothbrush varies according to age, and a population's characteristics. Various kinds of information must be provided for patients with periodontal diseases or those who poorly manage their oral hygiene. There is a lack of diversity in ultra-soft toothbrushes among commercially available toothbrushes, and data concerning the physical characteristics and clinical effect of ultra-soft tooth brushes or the safety of their use for plaque removal in the presence of injury are lacking. Therefore, this study measured the cleaning ability and abrasivity of ultra-soft toothbrushes and analyzed their effect according to bristle thickness to contribute to better toothbrush selection by customers and oral health promotion.

\section{Materials and Methods}

\section{Materials and subjects}

\section{1) Cleaning ability}

An ultra-soft toothbrush newly released by LINKO Co. (Seongnam, Korea) was used in this study. This ultrafine processed toothbrush has a bristle filament measuring 0.11 to $0.12 \mathrm{~mm}$ and bristle tip measuring less than $0.01 \mathrm{~mm}$ in thickness. Generally, soft toothbrushes have a filament thickness of 0.18 to $0.20 \mathrm{~mm}$ and tooth tip less than 0.01 $\mathrm{mm}$, and are $28 \%$ to $45 \%$ thicker than ultra-soft toothbrushes. This thickness difference has an influence on the degree of tooth abrasion and gingival stimulation. Bristle tips are processed into a tapered shape for easier insertion into the gingival sulcus. Due to the slim filament, bristles are densely arranged, soft, and elastic. Ultra-soft toothbrushes for children and periodontal diseases were selected, and differences in their cleaning ability according to age and function were investigated. The control group included toothbrushes that were the most sold toothbrushes in the three most visited online markets and had a bristle thickness of $0.2 \mathrm{~mm}$ or less. Ultimately, the Oral-B toothbrush for children and Aekyung 2080 original (Aekyung, Seoul, Korea) for periodontal diseases were selected. A gnathostatic model (Dentiform; Nissin Dental, Kyoto, Japan) and Arti-spray (Bausch, Hainspjitz, Germany) were used. An artificial plaque remover was used in the experiment.

\section{2) Abrasivity}

The same toothbrushes as those used in the cleaning ability experiment were used. For the abrasivity experiment, Perioe Sirintakhyo toothpaste (LG Household \& Health 
Care, Seoul, Korea), which has low abrasivity, was selected. The experiment was conducted using acrylic resin, Dentto-dam (Mediclus Co., Cheongju, Korea), and bovine teeth. Regarding experimental equipment, a toothbrush abrasivity measuring device (DE/D15 525X; Braun, Seoul, Korea), micro Vickers hardness tester (MMT-X7B; Matsuzawa, Akita, Japan), scanning electron microscopy (S-4700; Hithachi, Tokyo, Japan), and hard tissue cutting machine (Minitom; Struers, Ballerup, Denmark) were used.

\section{Method}

\section{1) Cleaning ability}

Arti-spray (wide-purpose color marker for testing occlusal contact or crown-bridge fit) was applied on the labial surface of the anterior region in the form of the gnathostatic model, and on the buccal surface of the posterior region to form artificial plaques. Arti-spray was sprayed once every five seconds at the same pressure and from the same location to ensure even application throughout dental surfaces. To investigate changes in the extent of dental plaque formation over time, the spraying time was changed after a five-second interval until the fifth spraying ${ }^{19)}$. Next, a toothbrush and a tooth, on which artificial dental plaques had formed, were fixed on the artificial dental plaque remover, and plaques on the buccal, lingual, and proximal surfaces (occlusal surface not included) were removed. Assuming that a person brushes each surface 10 times, the surfaces were brushed 10 times each at the same speed and pressure. The bristles and gnathostatic model were washed after each experiment. The Patient Hygiene Performance (PHP) index was used to determine the dental plaque index ${ }^{20,21)}$

\section{2) Abrasivity}

Bovine incisors with seemingly healthy surfaces were cut into $1 \mathrm{~cm} \times 1 \mathrm{~cm}$ pieces, and they were cast using resin. After the resin solidified, the resin block was removed from the cast, and cut such that the side opposite to the surface containing the bovine tooth was horizontally oriented. The resin layer was removed, and polished with silicon carbide paper to smooth the enamel surface. To test that the bovine tooth was appropriate for the experiment, the micro Vickers hardness test was used to measure the Vickers hardness number (VHN). The four corners of the enamel surface (upper left, upper right, lower left, and lower right) were pressed with a $200 \mathrm{~g}$ load for each corner. The pressed sites was measured at $400 \times$ magnification using a measuring microscope to measure the surface hardness of the enamel surface. Samples within the normal enamel surface hardness range of 280 to 380 VHN were selected. The samples were treated in preparation for scanning electron microscope (SEM). Since the samples were to be divided according to abrasion status, they were divided into a healthy enamel area and area to be brushed. Dentto-dam resins were applied to the areas that were not going to be brushed. A total of 12 samples were assigned to three on each groups according to the number of toothbrushing strokes (1,800 [2 months], 5,400 [6 months], 10,800 [12 months], and 21,600 [24 months]) to measure the extent of enamel abrasion. For the rolling toothbrushing method, it is recommended to brush each area at least 10 times. In general, teeth are based on brushing three times a day, and a total of 30 strokes are made in one tooth for one day. Based on this standard, 900 strokes would be made per month, and the subjects were assigned to groups based on this number. Next, the toothbrush abrasion tester was used to apply an appropriate number of toothbrushing strokes to the given tooth surface ${ }^{22)}$. After the experiment was over, Denttodam was removed, and the samples were treated through a standard procedure in preparation for SEM. They were analyzed at 5,000 $\times$ and $10,000 \times$ magnification at $10 \mathrm{kV}^{23}$.

\section{Statistical analysis}

To compare the results before and after the experiment on cleaning ability, the surface plaque index before and after toothbrushing was calculated using the PHP index for the control and experimental groups. A Shapiro-Wilk normality test was used to investigate plaque removal effectiveness. An independent t-test was performed if normality was confirmed. All statistical analyses were performed using IBM SPSS Statistics ver. 22.0 (IBM Co., Armonk, NY, USA) with the level of statistical significance set at $\mathrm{p}<0.05$. 


\section{Results}

\section{Cleaning ability}

To investigate differences in the level of plaque removal between soft and ultra-soft toothbrushes, normality and homoscedasticity tests were performed using the PHP indices of the control and experimental groups (Table 1). Analyzing toothbrushes for children and those for periodontal diseases showed that the level of plaque removal was on average 0.46 higher for the ultra-soft toothbrushes than the soft toothbrushes; however, a t-test found that the difference was not statistically significant (Table 2).

A normality test was performed to investigate differences in the level of plaque removal between the soft and ultra-soft toothbrushes for children and periodontal diseases. Normality was confirmed and a t-test was performed accordingly. In the case of toothbrushes for children, the level of plaque removal was on average 0.22 higher for the ultra-soft toothbrushes than the soft toothbrushes. Likewise, for the toothbrushes for periodontal diseases, the level of plaque removal was on average 0.59 higher for ultra-soft toothbrushes than soft toothbrushes. However, the differences were statistically non-significant in both

Table 1. Shapiro-Wilk Normality Test for Comparison of Patient Hygiene Performance (PHP) Index between the Control and Experimental Groups

\begin{tabular}{lccc}
\hline & Type of bristle & F & p-value \\
\hline PHP index & Ultra-soft & 0.942 & 0.409 \\
& Soft & 0.940 & 0.382 \\
\hline
\end{tabular}

Table 2. $\mathrm{t}$-test for Comparison of Patient Hygiene Performance (PHP) Index between All Experimental and Control Groups

\begin{tabular}{ccccccc}
\hline & \multicolumn{2}{c}{ Levene's test } & & \multicolumn{2}{c}{ t-test for equality of means } \\
\cline { 2 - 3 } \cline { 5 - 6 } & $\mathrm{F}$ & p-value & & $\mathrm{t}$ & p-value & $\begin{array}{c}\text { Mean } \\
\text { difference }\end{array}$ \\
\hline PHP index & 0.007 & 0.932 & & -1.338 & 0.192 & -0.46 \\
\hline
\end{tabular}

cases (Table 3).

\section{Abrasivity}

Differences in abrasivity were found in both the control and experimental groups in the SEM. Enamel surfaces that were performed to 1,800 brushing strokes showed smooth surfaces in some samples. However, they generally showed rough surfaces with deep grooves and hydroxyapatite crystals (Fig. 1A). On the other hand, abrasion was observed on enamel surfaces that were performed to the greatest number of strokes $(21,600)$; these surfaces were generally smooth with occasional shallow grooves (Fig. 1B). Based on this trend in which the enamel surface becomes smoother as the number of strokes increases, it was determined that toothbrush comparisons would be performed in the group with 21,600 strokes ( 24 months) as this group showed the greatest difference between before and after toothbrushing to determine abrasivity.

In the experiment using soft and ultra-soft toothbrushes for children, differences in the tooth surface between before and after toothbrushing, and therefore abrasivity, were observed in both groups. In the group that used the soft toothbrushes, tooth surfaces had deep groves and occasional dents before toothbrushing. However, the surfaces after toothbrushing had shallow grooves and were smooth overall (Fig. 2). In the group that used ultra-soft toothbrushes, tooth surfaces before toothbrushing exhibited hydroxyapatite crystals and shallow grooves. After toothbrushing, the hydroxyapatite crystals were partially removed and shallow grooves were observed on the tooth surface (Fig. 3). Therefore, the soft toothbrushes for children had higher abrasivity than the ultra-soft toothbrushes.

In the experiment using the soft and ultra-soft toothbrushes for periodontal diseases, differences in the tooth surface between before and after toothbrushing, and therefore abrasivity, were observed in both groups. In the

Table 3. t-test for Ultra-Soft and Soft Toothbrushes for Children and Periodontal Disease

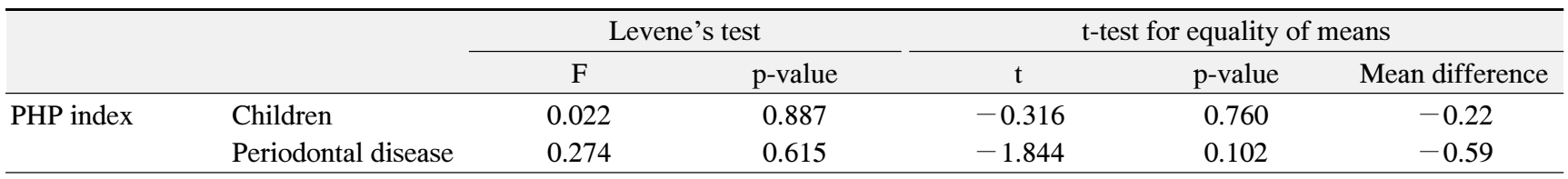

PHP: Patient Hygiene Performance. 

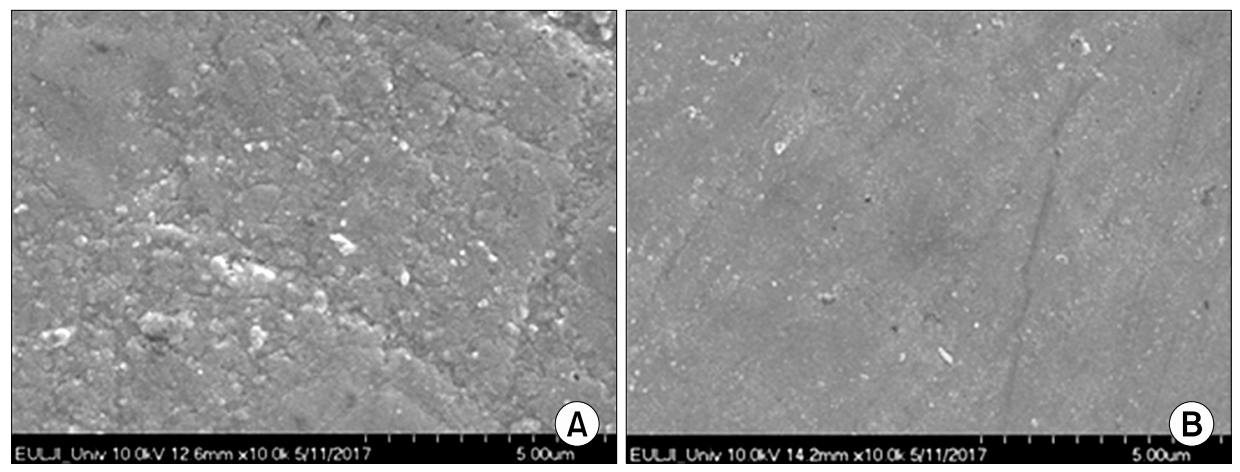

Fig. 1. (A) Number of brushing times: 1,800 . (B) Number of brushing times: 21,600 . The enamel surfaces subjected to 1,800 brush strokes had deep grooves and were rough due to crystallization of the apatite crystals. However, the surface of the enamel subjected to 21,600 brush strokes was observed to be smooth as a whole.
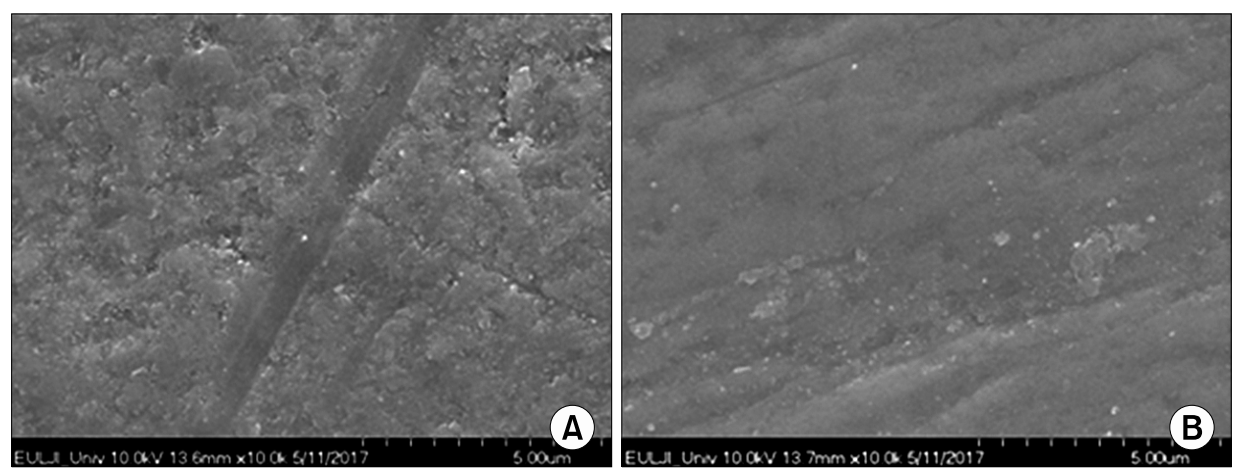

Fig. 2. Enamel surface before (A) and after (B) brushing with a toothbrush in children: The surface of the enamel after brushing was very shallow and smooth on the whole.
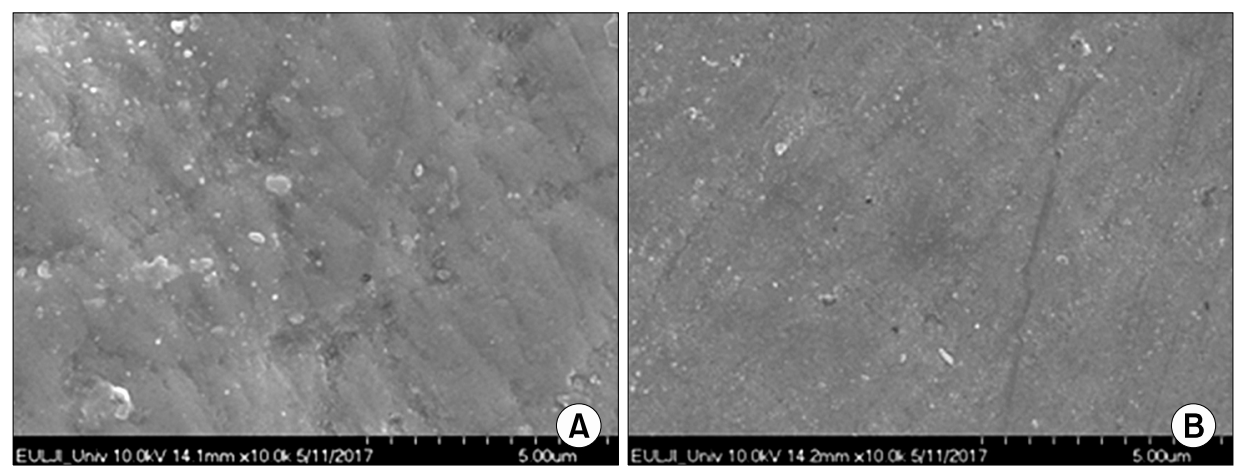

Fig. 3. Enamel surface before (A) and after (B) brushing with ultrasoft toothbrush in children: The surface of the enamel after brushing was partially cleared of hydroxyapatite crystals and overall a light groove was observed.

group that used the soft toothbrushes the tooth surfaces before toothbrushing were observed as very rough and cracks. After toothbrushing, the surface of tooth was observed very smooth as all removing cracks (Fig. 4). However, in the group that used the ultra-smooth toothbrushes, hydroxyapatite crystals and shallow grooves were observed on the tooth surfaces before toothbrushing. The hydroxyapatite crystals were partially removed after toothbrushing and the surfaces were generally smooth (Fig. 5). Therefore, the soft toothbrushes for periodontal diseases were shown to have higher abrasivity than the ultra-soft toothbrushes.

\section{Discussion}

Toothbrushing is the most general index of oral hygiene, it can affect the cleaning ability and abrasivity of a tooth surface depending on the hardness of the bristles. Dentists recommend regular toothbrushes or soft toothbrushes over hard toothbrushes, which can potentially damage the gingival and oral mucosa. However, customers choose bristle hardness based on their personal preference $^{24)}$. Although it is generally reported that soft toothbrush cause less abrasion than hard toothbrush ${ }^{25,26)}$, some studies have reported otherwise ${ }^{27,28)}$. They claim that soft toothbrush require a larger amount of toothpaste and 

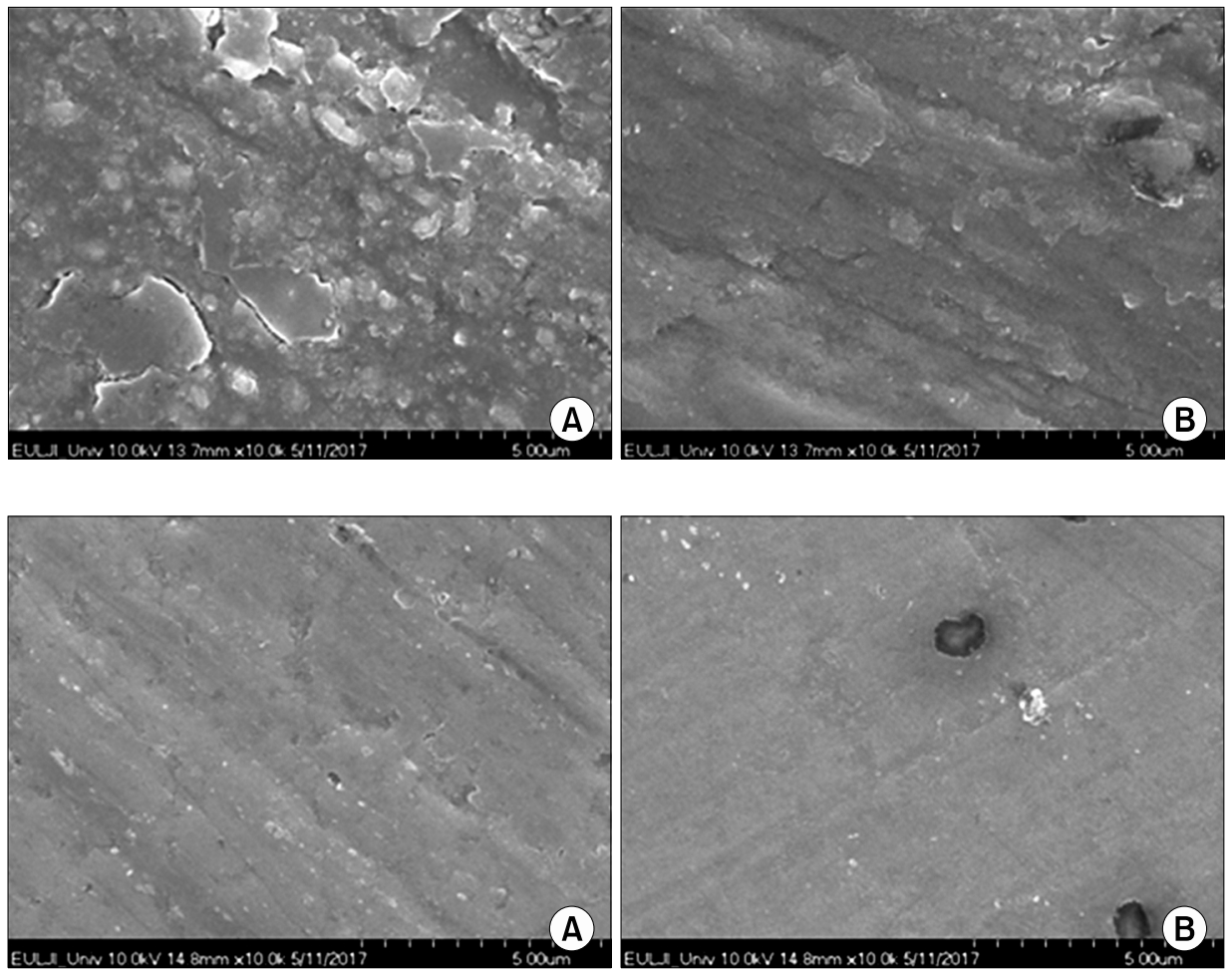

Fig. 4. Enamel surface before (A) and after (B) brushing with soft toothbrushes in individuals with periodontal disease: The surface of enamel after brushing was observed to be very smooth due to removal of cracks.

Fig. 5. Enamel surface before (A) and after (B) brushing using ultra-soft toothbrush in individuals with periodontal disease: The surface of the enamel after brushing was observed to have some grooves but was smooth overall. this increases the area of contact between the toothbrush and tooth surface, thereby increasing abrasivity. However, the increased area of contact also means a better cleaning ability. Therefore, it is necessary to develop soft toothbrushes that use a small amount of toothpaste to satisfy customer needs. This study was conducted to investigate the effect of newly released ultra-soft toothbrushes that can satisfy these needs. Cleaning ability and abrasion were compared between the ultra-soft toothbrushes, with the soft brushes as the control.

In the experiment on cleaning ability, the ultra-soft and soft toothbrushes did not show a statistically significant difference. In other words, their cleaning abilities were on a par with one another, or the ultra-soft toothbrushes have the same cleaning ability as the soft toothbrushes. Januar et al. ${ }^{9)}$ divided 65 students into two groups and conducted a double-blinded cross-over clinical trial to compare plaque removal effectiveness between toothbrushes with sharp bristles and those with round bristles. They also used the PHP index to assess their effectiveness. The group that used the toothbrushes with sharp bristles had a higher mean PHP index at seven days after the experiment. However, no significant difference in the mean PHP index was observed at 14 days. The bristles used in this study measured less than $0.01 \mathrm{~mm}$ in thickness and was sharp. Although direct comparison with the previous study is difficult since Januar et al. ${ }^{9)}$ did not measure bristle thickness, it can be inferred that bristle shape or diameter does not significantly affect cleaning ability. Agarwal et al. $^{29)}$, who investigated the effectiveness of different types of toothbrush, reported plaque indices measured from subjects who did not brush their teeth for 24 hours before coming to the dental clinic. Ultra-soft toothbrushes were found to have lower plaque indices compared with soft, regular, and hard toothbrushes, and thus had a better cleaning ability. As can be seen, the study results regarding the cleaning ability of toothbrushes greatly vary. If the limitation of the present study on bovine tooth is applied to a person in the future, it will be possible to provide data useful for the development of oral healthcare products.

In the abrasivity experiment, abrasivity increased in proportion to the number of brush strokes in two months (1,800 strokes) and 24 months (21,600 strokes) for both the ultra-soft and soft toothbrushes. In the 24 month toothbrushing experiment (21,600 strokes), the soft toothbrushes for children and those for periodontal diseases had 
higher abrasivity than the ultra-soft toothbrushes. This indicates that lower bristle diameters, or, in other words, softer bristles, and higher bristle density decrease abrasivity. Tellefsen et al. ${ }^{30)}$ studied the relationship between toothbrush bristle and surface roughness, and reported that as the number of filaments increases, surface roughness decreases, and vice versa. Wiegand et al. ${ }^{31)}$ reported that when the relative enamel abrasion of a toothpaste slurry with abrasion is 2.1 to 3.3 , a toothbrush with a filament diameter of $0.2 \mathrm{~mm}$ has higher enamel loss than that with a filament diameter of $0.15 \mathrm{~mm}$, meaning that abrasivity goes high with increases in filament diameter. Sasan et al. ${ }^{32}$ investigated whether commercially available toothbrushes had been classified according to the American Dental Association standard and recommended soft toothbrushes over hard toothbrushes since the latter can damage the tooth surface and the gingival margin, and since plaque removal depends more on the toothbrushing technique than on the bristle type. These findings support the results of this study.

The results of this study suggest that ultra-soft toothbrushes match the plaque removing ability of soft toothbrushes and reduce tooth abrasivity to minimize tooth damage. These results may arouse new interest in ultrasoft toothbrushes and may be useful in future research aimed at producing toothbrushes to be sold commercially. However, since the soft toothbrush used as the control in the cleaning ability experiment was a commercially available toothbrush, there were differences in the type of toothbrush, bristle number, length, and diameter. It was therefore difficult to systemically analyze the factors that can affect plaque removal. However, since a toothpaste with low abrasivity was used to control abrasion caused by toothpaste as has been done in a previous study ${ }^{30)}$, abrasion caused by toothpaste such as filament arrangement, density, and texture could be controlled ${ }^{27)}$. As ultra-soft toothbrushes have been shown to have lower abrasivity, use of these toothbrushes may help reduce customers' sensitivity by reducing tooth abrasion. By increasing the range of choices of toothbrushes tailored to individuals' tastes, the oral hygiene status of the nation, including the elderly population ${ }^{11)}$, may be improved.

\section{Acknowledgements}

This study was supported by research fund from Chosun University, 2017.

\section{References}

1. Petersen PE: Global policy for improvement of oral health in the 21 st century-implications to oral health research of World Health Assembly 2007, World Health Organization. Community Dent Oral Epidemiol 37: 1-8, 2009. https://doi.org/10.1111/j.1600-0528.2008.00448.x

2. Kim JB, Choi YJ, Back DI, et al.: Preventive dentistry. 5th ed. Komoonsa, Seoul, pp.6-13, 2009.

3. Löe $\mathrm{H}$ : Oral hygiene in the prevention of caries and periodontal disease. Int Dent J 50: 129-139, 2000. https://doi.org/10.1111/j.1875-595X.2000.tb00553.x

4. Collins FM: Toothbrush technology, dentifrices and dental biofilm removal. ADA CERP, Chicago, pp.1-11, 2009.

5. Ministry of Health and Welfare, Korea Health Promotion Foundation: Health plan 2020, 2016-2020. Ministry of Health and Welfare, Korea Health Promotion Foundation, Sejong, Seoul, pp.214-217, 2015.

6. Ministry of Health and Welfare: Health at a glance 2009 OECD indicators. Ministry of Health and Welfare, Sejong, pp.34-35, 2010.

7. Yankell SL: Toothbrushing and toothbrushing techniques. In: Harris NO, Christen AG, eds. Primary preventive dentistry. 3rd ed. Appleton and Lange, Norwalk, pp.79-106, 1991.

8. Shory NL, Mitchell GE, Jamison HC: A study of the effectiveness of two types of toothbrushes for removal of oral accumulations. J Am Dent Assoc 115: 717-720, 1987. https://doi.org/10.14219/jada.archive.1987.0310

9. Januar P, Susetyo A, Widyastuti R: The effectiveness of sharp end and rounded end bristle toothbrush. Dent J: Majalah Kedokteran Gigi 43: 122-125, 2010. https://doi.org/10.20473/j.djmkg.v43.i3.p122-125

10. International Organization for Standardization: Dentistry-Stiffness of the tufted area of tooth-brushes, ISO 8627:1987. International Organization for Standardization, Geneva, Switzerland, 1987.

11. Seo EJ, Shin SC, Seo HS, Kim EJ, Chang YS: A survey on Koreans' behavior about the use of oral hygiene devices. J 
Korean Acad Oral Health 27: 177-193, 2003.

12. Taylor H, Graham D, Cipak M, et al.: Brushing up on mouth care: an oral health resource for those who provide care to older adults. Dalhousie University, Halifax, pp.1-15, 2011.

13. Misra N, Maheshwari A, Misra P: Dental care in diabetes: a review. Indian J Public Health Res Dev 3: 102-105, 2012.

14. Montevecchi M, Moreschi A, Gatto MR, Checchi L, Checchi V: Evaluation of clinical effectiveness and subjective satisfaction of a new toothbrush for postsurgical hygiene care: a randomized split-mouth double-blind clinical trial. Scientific WorldJournal 2015: 828794, 2015. https://doi.org/10.1155/2015/828794

15. Ames NJ, Sulima P, Yates JM, et al.: Effects of systematic oral care in critically ill patients: a multicenter study. Am J Crit Care 20: e103-e114, 2011. https://doi.org/10.4037/ajcc2011359

16. Cherny NI, Fallon M, Kaasa S, Portenoy RK, Currow DC: Oxford textbook of palliative medicine. 5th ed. Oxford University Press, Oxford, pp.447-456, 2015.

17. Agnihotri R, Bhat GS, Bhat KM: Amlodipine-induced gingival overgrowth: considerations in a geriatric patient. Geriatr Gerontol Int 11: 365-368, 2011. https://doi.org/10.1111/j.1447-0594.2010.00659.x

18. Scattarella A, Petruzzi M, Ballini A, Grassi FR, Nardi GM: Oral lichen planus and dental hygiene: a case report. Int $\mathrm{J}$ Dent Hyg 9: 163-166, 2011. https://doi.org/10.1111/j.1601-5037.2010.00454.X

19. Lee $\mathrm{CH}$, Anh SH, Jang YH: An experimental study on plaque removal effect through the acting types of the electric toothbrushes. J Korean Soc Dent Hyg 11: 465-474, 2011.

20. Lim SH, Hwang JM: Relationship of oral health management behavior to plaque index systems. J Dent Hyg Sci 15: 159-165, 2015. https://doi.org/10.17135/jdhs.2015.15.2.159

21. Kim KE, Ahn E, Han JH: Variation in the index of dental plaque removal and practice assessment after instruction on toothbrushing. J Dent Hyg Sci 15: 220-225, 2015. https://doi.org/10.17135/jdhs.2015.15.2.220

22. Son JH, Lim DS, Ma DS, Park DY: Abrasion of resin infiltrated enamel by tooth brushing. J Korean Acad Oral
Health 37: 9-15, 2013.

https://doi.org/10.11149/jkaoh.2013.37.1.9

23. Han JS: Evaluation of air-powder abrasive system on tooth abrasion. J Korean Dent Assoc 28: 381-389, 1990.

24. Kyoizumi H, Yamada J, Suzuki T, Kanehira M, Finger WJ, Sasaki K: Effects of toothbrush hardness on in vitro wear and roughness of composite resins. J Contemp Dent Pract 14: 1137-1144, 2013. https://doi.org/10.5005/jp-journals-10024-1464

25. Harrington JH, Terry IA: Automatic and hand toothbrushing abrasions studies. J Am Dent Assoc 68: 343-350, 1964. https://doi.org/10.14219/jada.archive.1964.0099

26. Harte DB, Manly RS: Effect of toothbrush variables on wear of dentin produced by four abrasives. J Dent Res 54: 993-998, 1975. https://doi.org/10.1177/00220345750540054101

27. Dyer D, Addy M, Newcombe RG: Studies in vitro of abrasion by different manual toothbrush heads and a standard toothpaste. J Clin Periodontol 27: 99-103, 2000. https://doi.org/10.1034/j.1600-051x.2000.027002099.x

28. Wiegand A, Kuhn M, Sener B, Roos M, Attin T: Abrasion of eroded dentin caused by toothpaste slurries of different abrasivity and toothbrushes of different filament diameter. J Dent 37: 480-484, 2009. https://doi.org/10.1016/j.jdent.2009.03.005

29. Agarwal V, Agarwal S, Ranjan R: Effects of bristle hardness \& duration of manual tooth brushing on plaque control. Indian J Community Health 29: 123-128, 2017.

30. Tellefsen G, Liljeborg A, Johannsen A, Johannsen G: The role of the toothbrush in the abrasion process. Int J Dent Hyg 9: 284-290, 2011. https://doi.org/10.1111/j.1601-5037.2011.00505.x

31. Wiegand A, Schwerzmann M, Sener B, et al.: Impact of toothpaste slurry abrasivity and toothbrush filament stiffness on abrasion of eroded enamel: an in vitro study. Acta Odontol Scand 66: 231-235, 2008. https://doi.org/10.1080/00016350802195041

32. Sasan D, Thomas B, Mahalinga BK, Aithal KS, Ramesh PR: Toothbrush selection: a dilemma? Indian J Dent Res 17: 167-170, 2006. https://doi.org/10.4103/0970-9290.29869 